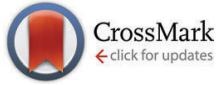

Cite this: J. Mater. Chem. C, 2016 , 4, 3640

\title{
Poly(methyl methacrylate)-grafted ZnO nanocomposites with variable dielectric constants by UV light irradiation
}

\begin{abstract}
Kenichi Hayashida*a and Yasuhiro Takatani ${ }^{\mathrm{b}}$
A series of poly(methyl methacrylate)-grafted $\mathrm{ZnO}$ nanoparticles (PMMA-ZnO) were synthesized using a surface-initiated polymerization technique and the optical and dielectric properties of PMMA-ZnO were studied. The dielectric constant $\varepsilon_{r}{ }^{\prime}$ of PMMA-ZnO thin films was highly increased by irradiation of UV light. It is indicated that electrons in the $\mathrm{ZnO}$ nanoparticles are excited from a valence band to a conductive band by absorption of UV light, resulting in a large increase in $\varepsilon_{r}{ }^{\prime}$ owing to Maxwell-Wagner polarization of the resultant free electrons. On the other hand, the dissipation factor $(\tan \delta)$ of PMMA$\mathrm{ZnO}$ is very low and almost constant during UV irradiation because PMMA-ZnO is electrically insulated by the grafted PMMA chains on the ZnO nanoparticles. Also, it was confirmed that due to the grafted PMMA chains, PMMA-ZnO nanocomposites exhibited low light scattering in addition to strong absorption of UV light. The low light scattering of PMMA-ZnO would enhance the absorption efficiency of UV light and therefore contribute to the large increase in $\varepsilon_{r}^{\prime}$ for PMMA-ZnO. Thus, PMMA-ZnO is a promising material for high sensitivity and low loss UV light sensors using the change in $\varepsilon_{r}^{\prime}$.
\end{abstract}

Received 1st March 2016, Accepted 29th March 2016

DOI: $10.1039 / \mathrm{c} 6 \mathrm{tc} 00882 \mathrm{~h}$

www.rsc.org/MaterialsC

\section{Introduction}

Dielectric materials are usually used to store electrical energy as capacitors. ${ }^{1}$ Among dielectric materials, polymer materials have the advantage of high flexibility, lightness, and processability although the very low dielectric constant $\varepsilon_{\mathrm{r}}{ }^{\prime}$ of polymer materials $\left(\varepsilon_{\mathrm{r}}{ }^{\prime}=2-3\right)$ strongly impedes improvement of the capacitance $C$ of capacitors. Because of the high $\varepsilon_{\mathrm{r}}{ }^{\prime}$ of ceramic materials $\left(\varepsilon_{\mathrm{r}}{ }^{\prime}>\right.$ 100), polymer/ceramic composite materials have been much developed over the last few decades. $\mathrm{BaTiO}_{3}$, one of the ferroelectric metal oxides, has been most widely used for enhancement of $\varepsilon_{\mathrm{r}}{ }^{\prime 2-10}$ On the other hand, for other dielectric applications such as variable capacitors, pressure sensors, and touch sensors, a change of $C$ for these systems is important, and high $\varepsilon_{\mathrm{r}}{ }^{\prime}$ is not always required. ${ }^{11-17}$ The distance between two electrodes $d$ and/or the area of the electrodes $S$ are varied by physical operations, changing the $C$ for the systems: $C=\varepsilon_{0} \varepsilon_{\mathrm{r}}{ }^{\prime} S / d$, where $\varepsilon_{0}$ is the permittivity of vacuum. Also, $C$ for a system can be changed when the $\varepsilon_{\mathrm{r}}{ }^{\prime}$ of a dielectric material is varied by a stimulus. For example, if the $\varepsilon_{\mathrm{r}}{ }^{\prime}$ of a polymer material could be changed by irradiation of light, the polymer material can be used not only for a variable capacitor but also for a light sensor.

\footnotetext{
${ }^{a}$ Materials and Processing Dept. II, Toyota Central R\&D Labs., Inc., Nagakute, Aichi 480-1192, Japan. E-mail: e1440@mosk.tytlabs.co.jp

${ }^{b}$ Materials Analysis and Evaluation Dept., Toyota Central R\&D Labs., Inc., Nagakute, Aichi 480-1192, Japan
}

However, no polymer material with a variable $\varepsilon_{\mathrm{r}}{ }^{\prime}$ by light has been reported so far.

Here, we suggest a composite system where semiconductor particles are incorporated in a polymer matrix. It is known that the conductivity of some semiconductors is strongly enhanced by absorption of light because electrons are excited from a valence band to a conductive band. ${ }^{18}$ Therefore, during light irradiation, the polymer/semiconductor composite should exhibit a high $\varepsilon_{\mathrm{r}}^{\prime}$ owing to Maxwell-Wagner polarization of the resultant free electrons. ${ }^{19}$ Unfortunately, it is expected that the dielectric loss of the composite simultaneously becomes very large owing to leak current by the free electrons. Generally, for polymer/inorganic particle composite systems, three-dimensional networks of inorganic particles are formed in the polymer matrix because interaction between the inorganic particles is stronger than that between the inorganic particles and the matrix polymer. ${ }^{9,20}$ For the polymer/semiconductor composite under light irradiation, therefore, the large leak current would be caused through the conductive networks of semiconductor particles. If the individual semiconductor particles are isolated and uniformly distributed in the polymer matrix, the composite should be electrically insulated to be a low-loss dielectric material. A successful method of insulation would be polymer grafting on the semiconductor particles. It has been reported that even carbon nanotubes with very high conductivity can be insulated by polymer grafting. ${ }^{21-23}$

In order to obtain a large $\varepsilon_{\mathrm{r}}{ }^{\prime}$ change $\Delta \varepsilon_{\mathrm{r}}{ }^{\prime}$ for the polymer/ semiconductor composite, it is desirable that the intrinsic $\varepsilon_{\mathrm{r}}{ }^{\prime}$ of 
the semiconductor is small enough in addition to high conductivity during light irradiation. The larger $\Delta \varepsilon_{\mathrm{r}}{ }^{\prime}$ provides better sensitivity of the light sensor. Furthermore, in terms of absorption efficiency of light for the composite, the smaller diameter of the semiconductor particles is desirable because of low scattering of light by the particles. Based on the above, $\mathrm{ZnO}$ is one of the most promising candidates for semiconductors with a variable $\varepsilon_{\mathrm{r}}{ }^{\prime}$. ZnO exhibits strong absorption of ultraviolet (UV) light, ${ }^{24,25}$ and the $\varepsilon_{\mathrm{r}}{ }^{\prime}$ of pure $\mathrm{ZnO}$ is as low as about $10 .^{26}$ In addition, a variety of $\mathrm{ZnO}$ nanoparticles are commercially and inexpensively available. $^{24,25}$

In this study, poly(methyl methacrylate) (PMMA) was grafted on ZnO nanoparticles using a surface-initiated atom transfer radical polymerization (SI-ATRP) technique. ${ }^{27}$ The optical and dielectric properties of the PMMA-grafted $\mathrm{ZnO}$ nanocomposites (PMMA-ZnO) were compared to those of conventional nanocomposites prepared by blending PMMA with the initiatormodified $\mathrm{ZnO}$ nanoparticles (PMMA/ZnO). We demonstrated that PMMA-ZnO exhibits a large $\Delta \varepsilon_{\mathrm{r}}{ }^{\prime}$ by irradiation of UV light with a low dissipation factor $(\tan \delta)$. To the best of our knowledge, this is the first report on polymer/semiconductor composites with a variable $\varepsilon_{\mathrm{r}}{ }^{\prime}$ by light irradiation although many studies have been reported on the dielectric properties of polymer/semiconductor composites such as polymer/ZnO composites. ${ }^{28-37}$

\section{Experimental}

\section{Sample preparation}

$\mathrm{ZnO}$ nanoparticles with an average diameter of $15 \mathrm{~nm}$ were obtained from Kanto Denka Kogyo (Japan). The density of the ZnO nanoparticles was determined to be $5.22 \mathrm{~g} \mathrm{~cm}^{-3}$ using an ultrapycnometer (UPY-2, Quantachrome). Procedures for modification of the ZnO nanoparticles were as follows (Fig. 1):

Amine-modification of the $\mathrm{ZnO}$ nanoparticles: trimethoxy[3(methylamino)propyl]silane $(840 \mu \mathrm{l}, 4.3 \mathrm{mmol})$ was added to the $\mathrm{ZnO}$ nanoparticles (10 g) homogeneously dispersed in dimethylformamide (DMF) $(97 \mathrm{ml})$ containing a small amount of water $(0.5 \mathrm{ml})$. The dispersion was sonicated and kept at $50{ }^{\circ} \mathrm{C}$ for $6 \mathrm{~h}$. The amine-modified $\mathrm{ZnO}$ nanoparticles (ZnO-NHMe) were purified by 3 cycles of centrifugation and redispersion in acetonitrile (AN).
Immobilization of ATRP initiators on the ZnO nanoparticles: p-(bromomethyl)benzyl 2-bromoisobutylate (BBnBiB) ${ }^{27}(1.0 \mathrm{~g}$, $2.9 \mathrm{mmol})$ and 1,8-bis(dimethylamino)naphthalene $(0.60 \mathrm{~g}$, $2.8 \mathrm{mmol}$ ) were added to $\mathrm{ZnO}-\mathrm{NHMe}(16 \mathrm{~g})$ dispersed in AN $(95 \mathrm{ml})$. The mixture was sonicated and kept at $40{ }^{\circ} \mathrm{C}$ for $12 \mathrm{~h}$. The initiator-modified $\mathrm{ZnO}$ nanoparticles $(\mathrm{ZnO}-\mathrm{Br})$ were purified by 3 cycles of centrifugation and redispersion in DMF.

SI-ATRP from the $\mathrm{ZnO}$ nanoparticles: the following is an example. $N, N$-Dimethylacetamide $(90 \mathrm{ml})$ was added to $\mathrm{ZnO}-\mathrm{Br}$ $(16.8 \mathrm{~g})$ and $\mathrm{CuBr}(14.3 \mathrm{mg}, 100 \mu \mathrm{mol})$ in a $\mathrm{N}_{2}$ atmosphere. After sonication of the mixture, 2,2'-bipyridyl $(46.8 \mathrm{mg}, 300 \mu \mathrm{mol})$ in methyl methacrylate $(60 \mathrm{ml})$ was added, and the dispersion was kept at $60{ }^{\circ} \mathrm{C}$ for $2.5 \mathrm{~h}$. The resultant core-shell nanoparticles (PMMA-ZnO) were precipitated in methanol and freeze-dried by 1,4-dioxane.

PMMA/ZnO nanocomposites were prepared by blending $\mathrm{ZnO}-\mathrm{Br}$ with PMMA. First, $\mathrm{ZnO}-\mathrm{Br}$ and the PMMA matrix were added to 1,4-dioxane and homogeneously dispersed by sonication. The dispersion was quickly frozen in liquid nitrogen, and then freeze-dried under vacuum. Subsequently, the preliminary mixed PMMA/ZnO nanocomposites were further kneaded in a molten state. Thus-obtained PMMA/ZnO and PMMA-ZnO nanocomposites were molded into disk-like specimens with a diameter of $33 \mathrm{~mm}$ and a thickness of $\sim 0.52 \mathrm{~mm}$ by compression at $120-140{ }^{\circ} \mathrm{C}$.

\section{Measurements}

The ZnO contents in PMMA/ZnO and PMMA-ZnO were determined by thermogravimetry (TG). The sample taken in a platinum pan was heated from room temperature to $600{ }^{\circ} \mathrm{C}$ at a rate of $10{ }^{\circ} \mathrm{C} \mathrm{min}^{-1}$ under an air flow $\left(200 \mathrm{ml} \mathrm{min}^{-1}\right)$ using a thermobalance (Thermo Plus TG 8120, Rigaku). The volume fraction of $\mathrm{ZnO} \Phi_{\mathrm{ZnO}}$ for PMMA/ZnO and PMMA-ZnO was calculated using a density of $5.22 \mathrm{~g} \mathrm{~cm}^{-3}$ for the $\mathrm{ZnO}$ nanoparticles (the residue after TG measurement) and of $1.19 \mathrm{~g} \mathrm{~cm}^{-3}$ for the organic components (the weight loss during the TG measurement).

SEM images of the modified $\mathrm{ZnO}$ nanoparticles were obtained using an SEM (S-5500, Hitachi) operated at an accelerating voltage of $1 \mathrm{kV}$. The cross-section of the molded samples that had been flattened with the argon ion beam using a cross section polisher ${ }^{38}$ was observed at an accelerating voltage of $2 \mathrm{kV}$.

Transmission spectra of the disk-like specimens were obtained using a UV-vis spectrophotometer (UV-3600, Shimadzu),

(a)

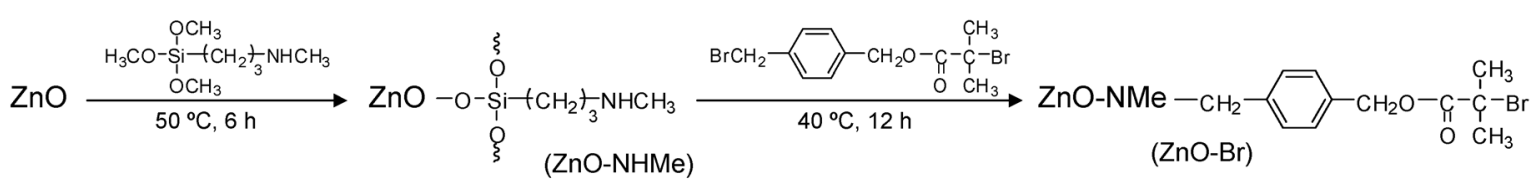

(b)

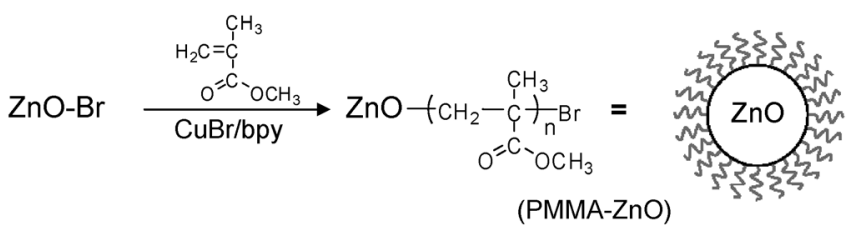

Fig. 1 Synthesis procedure of poly(methyl methacrylate)-grafted $\mathrm{ZnO}$ particles (PMMA-ZnO). (a) Preparation of ATRP initiator-modified ZnO particles ( $\mathrm{ZnO}-\mathrm{Br}$ ). (b) Surface-initiated ATRP of methyl methacrylate using $\mathrm{ZnO}-\mathrm{Br}$. 


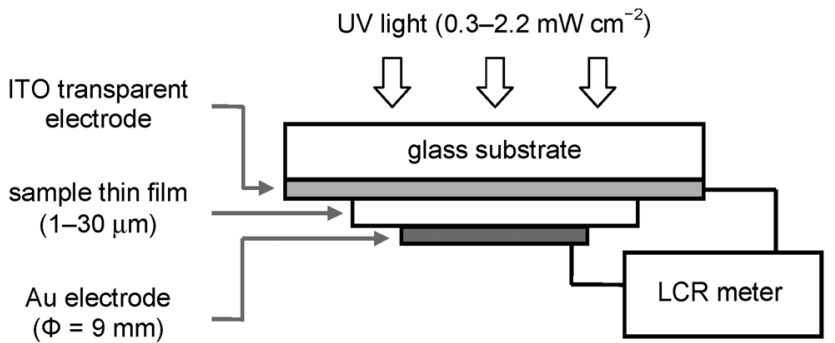

Fig. 2 Experimental set-up for dielectric measurements under UV light irradiation at $365 \mathrm{~nm}$.

where the sample was scanned in the wavelength $\lambda$ range of $300-800 \mathrm{~nm}$ at a resolution of $2 \mathrm{~nm}$.

Complex permittivity of the disk-like specimen was recorded in the frequency range of $10^{2}-10^{6} \mathrm{~Hz}$ using an LCR meter (E4980A, Agilent) operated at $2 \mathrm{~V}$, where two gold electrodes with a diameter of $27 \mathrm{~mm}$ were deposited on the top and bottom of the specimens. In the case of dielectric measurements under UV light irradiation, a thin film of the sample $(1-30 \mu \mathrm{m})$ was formed from $\gamma$-butyrolactone solution using spin-coating on an indium tin oxide (ITO) electrode supported by a glass substrate as shown in Fig. 2. The values of capacitance and $\tan \delta$ at $1 \mathrm{kHz}$ were measured after the UV irradiation for 3 min using an LCR meter (IM3523, Hioki) operated at $2 \mathrm{kV}$. The film thickness was calculated using the capacitance of the thin film and the obtained $\varepsilon_{\mathrm{r}}{ }^{\prime}$ for the corresponding disk-like specimen. UV intensity was changed in the range of $0.3-2.2 \mathrm{~mW} \mathrm{~cm}^{-2}$ by varying the distance between the UV lamp (SLUV-4, AS ONE) and the sample. The actual UV intensity was measured using a UV light meter (UV-340C, CUSTOM).

\section{Results and discussion}

\section{Sample preparation and characterization}

ATRP-initiator modified $\mathrm{ZnO}$ nanoparticles $(\mathrm{ZnO}-\mathrm{Br})$ were synthesized as shown in Fig. 1a, where the $\mathrm{ZnO}$ nanoparticles with an average diameter of $15 \mathrm{~nm}$ were used. In the first step, amine-functionalized $\mathrm{ZnO}$ nanoparticles (ZnO-NHMe) were prepared using a silane coupling reagent containing an aliphatic amine group. In the second step, ZnO-NHMe was reacted with an ATRP initiator with a benzyl bromide structure (BBnBiB), ${ }^{17}$ and the ATRP-initiator moiety was introduced on the $\mathrm{ZnO}$ surface (ZnO-Br). Methyl methacrylate was polymerized from $\mathrm{ZnO}-\mathrm{Br}$, and PMMA chains were grafted on the ZnO nanoparticles (PMMA-ZnO) as shown in Fig. 1b. Four kinds of coreshell nanoparticles with various PMMA chain lengths were synthesized as listed in Table 1 . The volume fraction of $\mathrm{ZnO} \Phi_{\mathrm{ZnO}}$ for PMMA-ZnO was calculated using TG results (Fig. 3). Also, the grafting of the PMMA chains was recognized by high resolution SEM images in Fig. 4. After the grafting, the diameter of the coreshell nanoparticles becomes much larger than before.

Conventional nanocomposites (PMMA/ZnO) were also prepared by blending the $\mathrm{ZnO}-\mathrm{Br}$ nanoparticles with PMMA, and designed to have the same $\Phi_{\mathrm{Zno}}$ as PMMA-ZnO. The two types of nanocomposite samples were molded by hot pressing. The dispersivity of the $\mathrm{ZnO}$ nanoparticles in the molded specimens was observed
Table 1 Characteristics of PMMA/ZnO and PMMA-ZnO composites

\begin{tabular}{lll}
\hline Sample & $\Phi_{\mathrm{Zno}}{ }^{a}$ & PMMA shell thickness ${ }^{b}[\mathrm{~nm}]$ \\
\hline PMMA/ZnO & 0.054 & - \\
& 0.105 & - \\
& 0.173 & - \\
PMMA-ZnO & 0.239 & - \\
& 0.051 & 13 \\
& 0.107 & 8.2 \\
& 0.172 & 6.0 \\
0.233 & 4.7
\end{tabular}

${ }^{a}$ Calculated using a density of $5.22 \mathrm{~g} \mathrm{~cm}^{-3}$ for the $\mathrm{ZnO}$ nanoparticles and of $1.19 \mathrm{~g} \mathrm{~cm}^{-3}$ for the organic components. ${ }^{b}$ Calculated from the average diameter of the $\mathrm{ZnO}$ nanoparticles $(15 \mathrm{~nm})$ and $\Phi_{\mathrm{ZnO}}$.

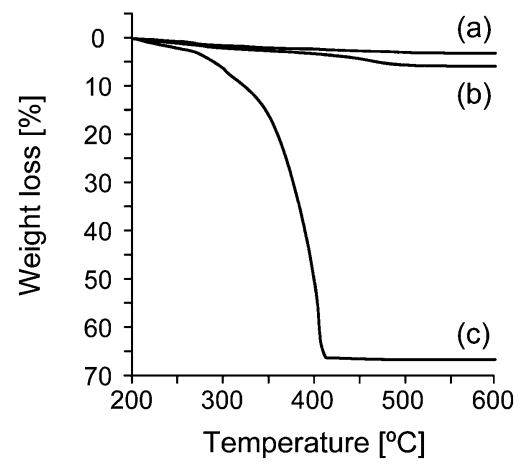

Fig. 3 TG curves of (a) $\mathrm{ZnO}$, (b) $\mathrm{ZnO}-\mathrm{Br}$, and (c) PMMA-ZnO with $\Phi_{\mathrm{ZnO}}=$ 0.11 in an air flow.

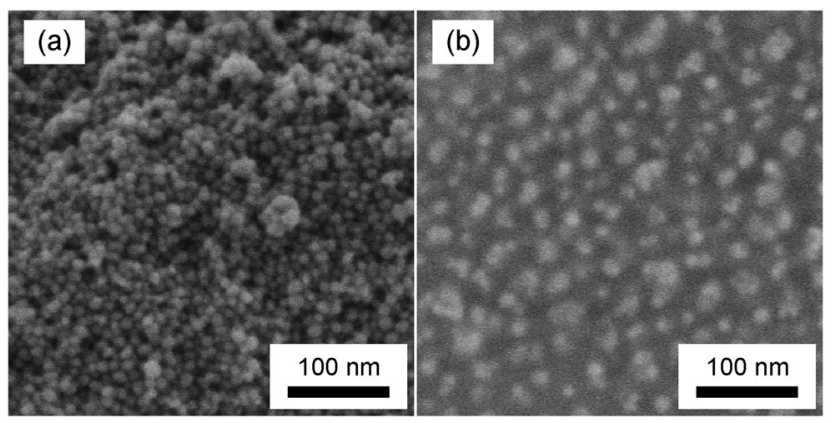

Fig. 4 High resolution SEM images of modified $\mathrm{ZnO}$ nanoparticles. (a) $\mathrm{ZnO}-\mathrm{Br}$. (b) PMMA-ZnO with $\Phi_{\mathrm{ZnO}}=0.11$.

by SEM. Fig. 5 shows SEM images of the two types of nanocomposites with $\Phi_{\mathrm{ZnO}}=0.11$. For PMMA/ZnO, the $\mathrm{ZnO}$ nanoparticles are inhomogeneously dispersed and form agglomerates with a submicron size. In contrast, the dispersivity of the $\mathrm{ZnO}$ nanoparticles is surprisingly good for PMMA-ZnO. In the PMMA-ZnO system, the ZnO nanoparticles are forced to be isolated and uniformly dispersed by the grafted PMMA shells.

\section{Optical and dielectric properties of the molded samples}

The difference in dispersivity of the $\mathrm{ZnO}$ nanoparticles between the two types of nanocomposites strongly influences light transmittance $T$. Fig. 6 shows the transmission spectra of the two types of nanocomposites in the $\lambda$ range of $300-800 \mathrm{~nm}$. Even at $\Phi_{\mathrm{ZnO}}=0.05, \mathrm{PMMA} / \mathrm{ZnO}$ exhibits almost zero $T$ in the 


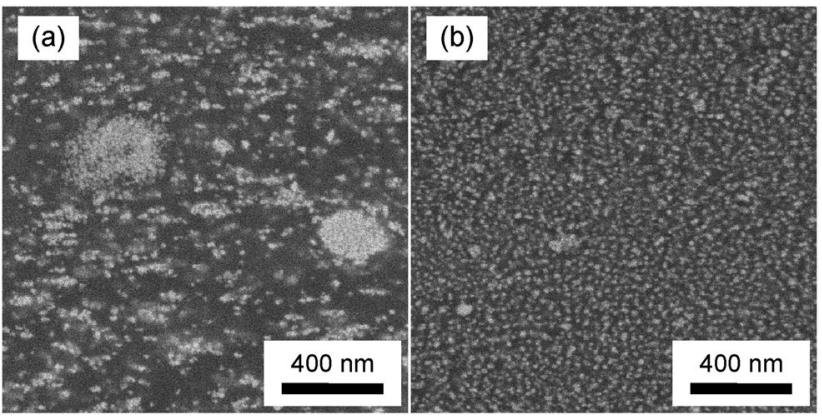

Fig. 5 SEM images of cross-sections of two types of composites with $\Phi_{\mathrm{ZnO}}=0.11$. (a) PMMA/ZnO. (b) PMMA-ZnO.

overall $\lambda$ range. This is because the inhomogeneity of PMMA/ $\mathrm{ZnO}$ causes intensive scattering of light. In contrast, PMMA-ZnO exhibits high transparency in the $\lambda$ range of $400-800 \mathrm{~nm}$ in spite of the large thickness of $\sim 0.52 \mathrm{~mm}$. The relatively low transparency in the lower $\lambda$ range $(400-600 \mathrm{~nm})$ would be due to light scattering by the individual $\mathrm{ZnO}$ nanoparticles rather than absorption by the $\mathrm{ZnO}$ nanoparticles. On the other hand, almost zero $T$ at less than $400 \mathrm{~nm}$ must be mainly attributed to absorption by the $\mathrm{ZnO}$ nanoparticles. According to the literature, $\mathrm{ZnO}$ has a wide band gap of $3.2 \mathrm{eV}^{18}$ and therefore absorbs UV light less than $390 \mathrm{~nm}$.

For dielectric measurements, gold electrodes were deposited on the molded specimens by sputter coating. In Fig. $7, \varepsilon_{\mathrm{r}}{ }^{\prime}$ and $\tan \delta$ of the two types of nanocomposites at $1 \mathrm{kHz}$ are plotted as a function of $\Phi_{\mathrm{Zno}}$. Because of the low $\varepsilon_{\mathrm{r}}{ }^{\prime}$ of $\mathrm{ZnO}\left(\varepsilon_{\mathrm{r}}{ }^{\prime} \approx 10\right),{ }^{26}$ both the nanocomposites have relatively low $\varepsilon_{\mathrm{r}}{ }^{\prime} \mathrm{s}$ in comparison with $\mathrm{PMMA} / \mathrm{BaTiO}_{3}$ composites. ${ }^{4,8,9}$ In addition, PMMA-ZnO has a smaller $\varepsilon_{\mathrm{r}}{ }^{\prime}$ than PMMA/ZnO, resulting from the better dispersivity of the ZnO nanoparticles for PMMA-ZnO. The same phenomenon has already been reported for a $\mathrm{PMMA} / \mathrm{BaTiO}_{3}$ composite system, and a detailed explanation is provided. ${ }^{9}$ Moreover, Wang and Tan have also demonstrated that a composite

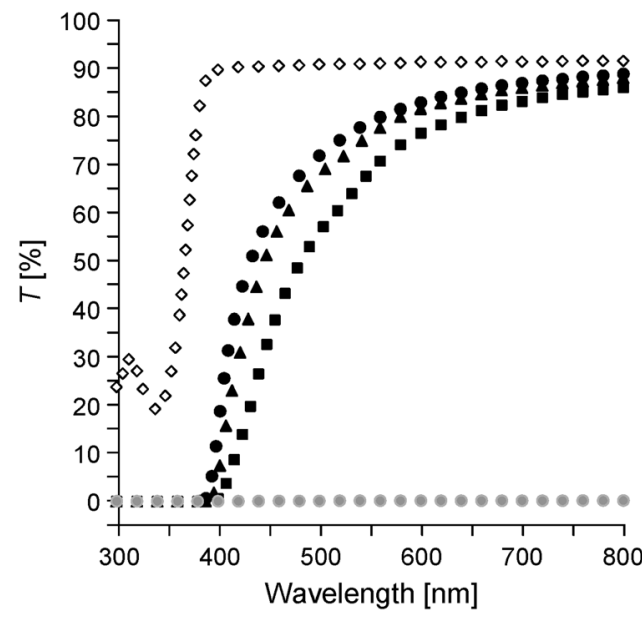

Fig. 6 Transmission spectra of PMMA (diamonds) and two types of composites with $\Phi_{\mathrm{ZnO}}=0.05$ (circles), $\Phi_{\mathrm{ZnO}}=0.11$ (triangles), and $\Phi_{\mathrm{ZnO}}=0.23$ (squares). Gray symbols, PMMA/ZnO; black symbols, PMMA-ZnO. The thickness of the specimens is around $0.52 \mathrm{~mm}$.

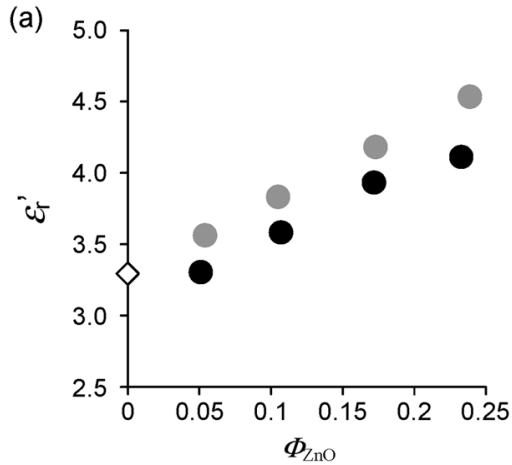

(b)

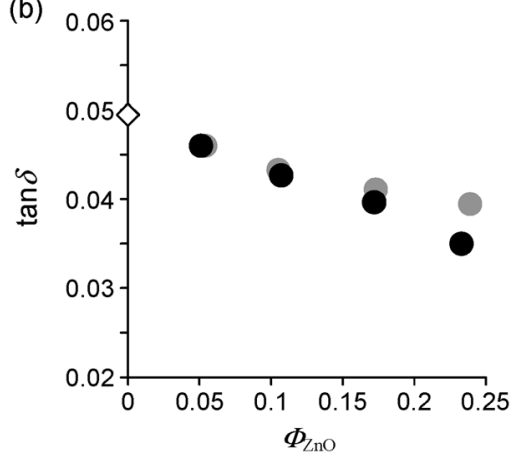

Fig. 7 (a) Dielectric constant $\varepsilon_{\mathrm{r}}{ }^{\prime}$ and (b) dissipation factor ( $\tan \delta$ ) of PMMA (diamonds) and two types of composites at $1 \mathrm{kHz}$ as a function of $\Phi_{\mathrm{ZnO}}$. Gray circles, PMMA/ZnO; black circles, PMMA-ZnO.

with agglomerated particles exhibited slightly higher $\varepsilon_{\mathrm{r}}{ }^{\prime}$ than that with well-dispersed particles using a simulation method. ${ }^{39}$

Dielectric properties of the thin samples during UV light irradiation

As mentioned above, PMMA-ZnO is a promising material for UV light sensors using $\Delta \varepsilon_{\mathrm{r}}{ }^{\prime}$ because of the strong absorption of UV light, low light scattering, and relatively low $\varepsilon_{\mathrm{r}}^{\prime}$. To demonstrate the potential for the capacitive UV light sensor, we set up a dielectric measurement system under UV light irradiation as shown in Fig. 2. In this measurement system, in order to irradiate UV light effectively on the sample, a transparent electrode made of ITO was used. The thickness of the sample was designed to be 1-30 $\mu \mathrm{m}$ using spincoating from $\gamma$-butyrolactone solution. For the spin-coating of PMMA-ZnO, $\gamma$-butyrolactone was the best among general purpose solvents such as toluene, chloroform, tetrahydrofuran and DMF, which gave a clear and smooth thin film coated on the transparent ITO electrode. Fig. 8 shows $\varepsilon_{\mathrm{r}}^{\prime}$ and $\tan \delta$ of the PMMA-ZnO thin films with $\Phi_{\mathrm{ZnO}}=0.17$ at $1 \mathrm{kHz}$ after UV light irradiation. As the UV light intensity becomes strong, the $\varepsilon_{\mathrm{r}}^{\prime}$ of PMMA-ZnO is highly increased. It is indicated that by absorption of UV light, electrons in the $\mathrm{ZnO}$ nanoparticles are excited from a valence band to a conductive band, ${ }^{18}$ resulting in large $\Delta \varepsilon_{\mathrm{r}}{ }^{\prime}$ owing to Maxwell-Wagner polarization of the resultant free electrons. ${ }^{19}$ In addition, the $\varepsilon_{\mathrm{r}}{ }^{\prime}$ of the PMMA-ZnO thin film becomes lower as the thickness is larger, suggesting that a thickness over $1 \mu \mathrm{m}$ is too large for UV light to penetrate into the inside of the PMMA-ZnO thin films because UV light is strongly absorbed by the $\mathrm{ZnO}$ nanoparticles. Although we also tried to prepare PMMA-ZnO thin films with a thickness less than $1 \mu \mathrm{m}$, the reproducibility of the dielectric 

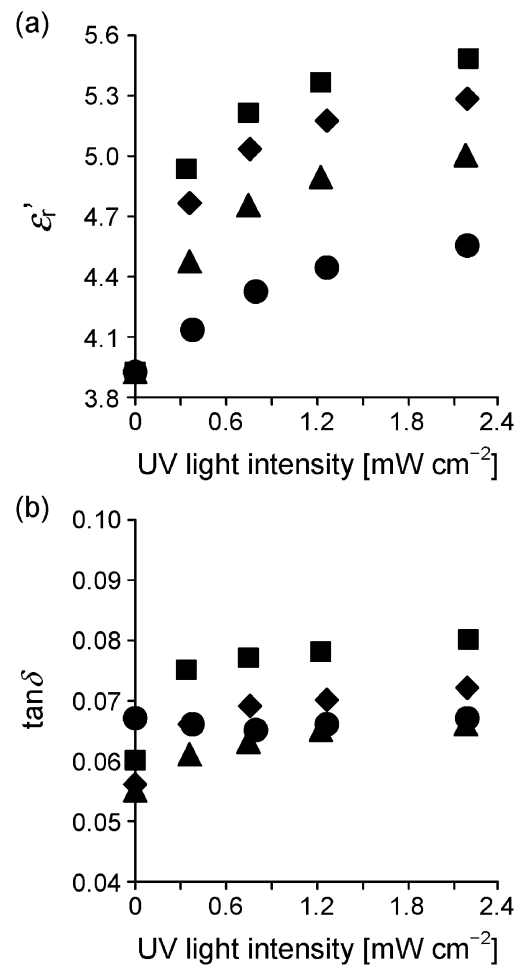

Fig. 8 (a) Dielectric constant $\varepsilon_{r}{ }^{\prime}$ and (b) $\tan \delta$ of PMMA-ZnO with $\Phi_{\text {ZnO }}=$ 0.17 at $1 \mathrm{kHz}$ as a function of UV light intensity. The thickness of the $\mathrm{PMMA}-\mathrm{ZnO}$ thin films is calculated to be $11 \mu \mathrm{m}$ (circles), $4.2 \mu \mathrm{m}$ (triangles), $2.0 \mu \mathrm{m}$ (diamonds), and $1.0 \mu \mathrm{m}$ (squares).

properties was poor probably because the thickness was excessively inhomogeneous. On the other hand, the $\tan \delta$ of PMMA-ZnO is very low and almost constant as shown in Fig. 8b. This is because PMMA-ZnO is electrically insulated by the grafted PMMA chains on the ZnO nanoparticles. The ideal thickness of the PMMA shell is calculated from $\Phi_{\mathrm{ZnO}}$ and the average diameter of the $\mathrm{ZnO}$ nanoparticles, and is listed in Table 1. The thick PMMA shell over $5 \mathrm{~nm}$ would prevent tunneling conduction between the $\mathrm{ZnO}$ nanoparticles by the free electrons according to an impedance analysis reported before. ${ }^{22}$ In this dielectric measurement, a PMMA/ZnO thin film with $\Phi_{\mathrm{ZnO}}=0.17$ was also prepared, and the dielectric properties under UV light irradiation were evaluated in the same manner. During UV light irradiation with an intensity of $2.2 \mathrm{~mW} \mathrm{~cm}^{-2}$, the $\tan \delta$ of the PMMA/ZnO thin film was over 10 which was out of the range of the used LCR meter. This extremely large dielectric loss is due to leak current by the free electrons.

Fig. 9 shows dielectric properties of PMMA-ZnO thin films with a thickness of about $1 \mu \mathrm{m}$ at $1 \mathrm{kHz}$ after UV irradiation with an intensity of $2.2 \mathrm{~mW} \mathrm{~cm}{ }^{-2}$. The increase rate for the $\varepsilon_{\mathrm{r}}{ }^{\prime}$ of PMMA-ZnO after UV irradiation $R$ was calculated by the following equation: $R=\Delta \varepsilon_{\mathrm{r}}{ }^{\prime} / \varepsilon_{\mathrm{r}}{ }^{\prime}$, where $\varepsilon_{\mathrm{r}}{ }^{\prime}$ is the $\varepsilon_{\mathrm{r}}{ }^{\prime}$ of PMMA$\mathrm{ZnO}$ before UV irradiation. $\Delta \varepsilon_{\mathrm{r}}{ }^{\prime} / \varepsilon_{\mathrm{r}}{ }_{0}^{\prime}$ is more than $10 \%$ when $\Phi_{\mathrm{ZnO}}>0.1$, which is large enough for the capacitive UV light sensor. Furthermore, the $\tan \delta$ of PMMA-ZnO is less than 0.12 as shown in Fig. $9 \mathrm{~b}$ although the $\tan \delta$ value is increased when $\Phi_{\mathrm{ZnO}}>0.1$. These results demonstrate that PMMA-ZnO has a high sensitivity and a low dielectric loss for the capacitive UV light sensor.
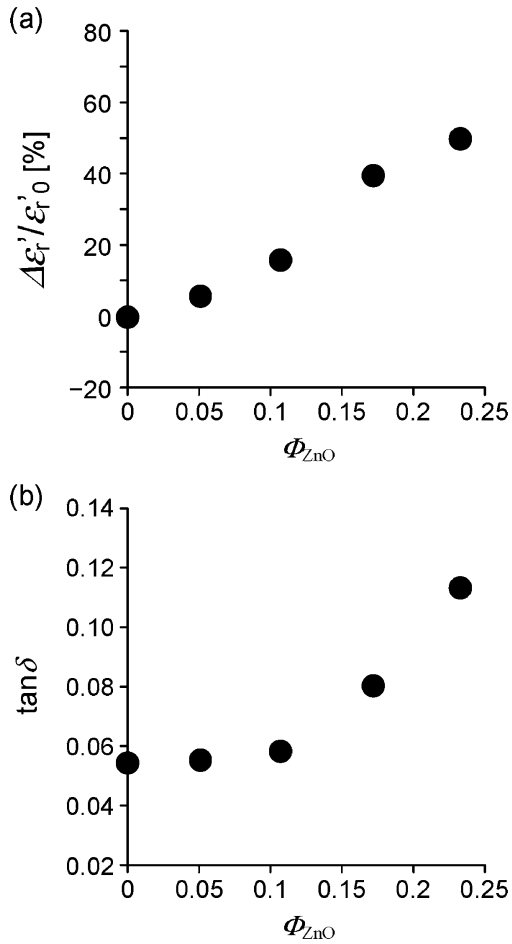

Fig. 9 Dielectric properties of PMMA-ZnO thin films with a thickness of about $1 \mu \mathrm{m}$ at $1 \mathrm{kHz}$ as a function of $\Phi_{Z n O}$ after UV irradiation with an intensity of $2.2 \mathrm{~mW} \mathrm{~cm}{ }^{-2}$. (a) Increase rate for $\varepsilon_{\mathrm{r}}{ }^{\prime}$ of PMMA-ZnO. The $\varepsilon_{\mathrm{r}}{ }^{\prime}$ change $\Delta \varepsilon_{\mathrm{r}}{ }^{\prime}$ is normalized by the $\varepsilon_{\mathrm{r}}{ }^{\prime}$ before UV irradiation $\varepsilon_{\mathrm{r}}{ }^{\prime}$. (b) $\tan \delta$ of PMMA-ZnO.

For the application of a UV light sensor, the response speed of $\varepsilon_{\mathrm{r}}{ }^{\prime}$ is also important. Fig. 10 shows the response speed of the $\varepsilon_{\mathrm{r}}{ }^{\prime}$ of the PMMA-ZnO thin film with $\Phi_{\mathrm{ZnO}}=0.23$ to UV irradiation with various intensities. The $\varepsilon_{\mathrm{r}}$ 's are sharply increased right after UV irradiation and saturated in a few minutes during UV irradiation. When UV light turns off, the increased $\varepsilon_{\mathrm{r}}$ 's are relaxed to the initial value in about 20 minutes. Owing to the low response speed for UV light irradiation, we suggest that the PMMA-ZnO thin films are used

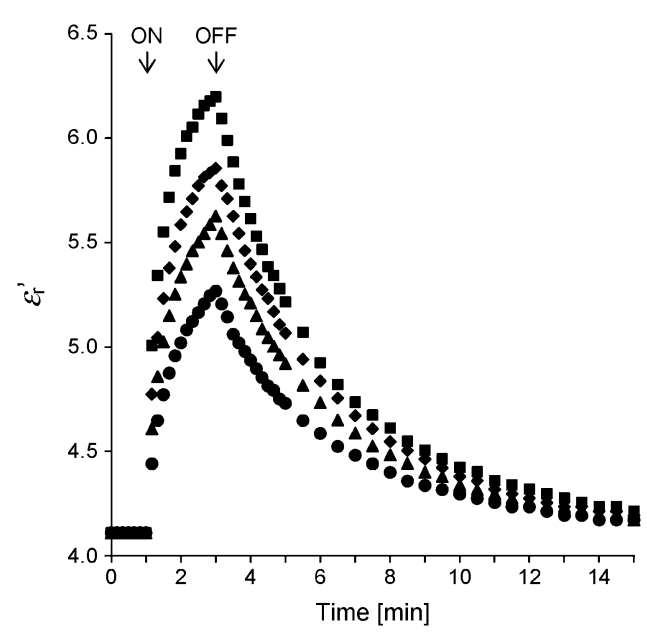

Fig. 10 Response of $\varepsilon_{r}{ }^{\prime}$ at $1 \mathrm{kHz}$ to $U \mathrm{~V}$ irradiation with various intensities of $0.4 \mathrm{~mW} \mathrm{~cm}^{-2}$ (circles), $0.8 \mathrm{~mW} \mathrm{~cm}^{-2}$ (triangles), $1.3 \mathrm{~mW} \mathrm{~cm}^{-2}$ (diamonds), and $2.2 \mathrm{~mW} \mathrm{~cm}^{-2}$ (squares) for PMMA-ZnO with $\Phi_{\mathrm{ZnO}}=0.23$. The thickness of the PMMA-ZnO thin film is $1.1 \mu \mathrm{m}$. 
for a sunlight sensor that controls outdoor illumination for which no quick response is required. Because the UV intensity in sunlight is around $3 \mathrm{~mW} \mathrm{~cm}^{-2}$, PMMA-ZnO should have a sufficient sensitivity for sunlight. In fact, we have experimentally confirmed that PMMA-ZnO had a good sensitivity for sunlight. Further investigation on the capacitive UV light sensor using PMMA-ZnO is in progress.

\section{Conclusion}

In summary, a dielectric constant $\varepsilon_{\mathrm{r}}{ }^{\prime}$-variable material with low dielectric loss was produced using polymer-grafted $\mathrm{ZnO}$ nanoparticles. In this study, a series of poly(methyl methacrylate)grafted $\mathrm{ZnO}$ nanoparticles (PMMA-ZnO) were synthesized using a surface-initiated polymerization technique. The $\varepsilon_{\mathrm{r}}{ }^{\prime}$ of PMMA-ZnO thin films was highly increased by irradiation of UV light. It is indicated that electrons in the $\mathrm{ZnO}$ nanoparticles are excited from a valence band to a conductive band by absorption of UV light, resulting in a highly increased $\varepsilon_{\mathrm{r}}{ }^{\prime}$ owing to Maxwell-Wagner polarization of the resultant free electrons. On the other hand, the dissipation factor $(\tan \delta)$ of PMMA-ZnO is very low and almost constant during UV irradiation because PMMA-ZnO is electrically insulated by the grafted PMMA chains on the ZnO nanoparticles. Also, it was confirmed that due to the grafted PMMA chains, PMMA-ZnO nanocomposites exhibited low light scattering in addition to strong absorption of UV light. The low light scattering of PMMA-ZnO would enhance the absorption efficiency of UV light and therefore contribute to the large increase in $\varepsilon_{\mathrm{r}}^{\prime}$ for PMMA-ZnO. Thus, PMMA-ZnO is a promising material for high sensitivity and low loss UV light sensors using the change in $\varepsilon_{\mathrm{r}}{ }^{\prime}$.

\section{References}

1 W. J. Sarjeant, J. Zirnheld, F. W. MacDougall, J. S. Bowers, N. Clark, I. W. Clelland, R. A. Price, M. Hudis, I. Kohlberg, G. McDuff, I. McNab, S. G. Parler Jr and J. Prymak, in Handbook of Low and High Dielectric Constant Materials and Their Applications, ed. H. S. Nalwa, Academic Press, London, 1999, vol. 2, ch. 9, pp. 423-491.

2 P. Kim, S. C. Jones, P. J. Hotchkiss, J. N. Haddock, B. Kippelen, S. R. Marder and J. W. Perry, Adv. Mater., 2007, 19, 1001-1005.

3 H. M. Jung, J.-H. Kang, S. Y. Yang, J. C. Won and Y. S. Kim, Chem. Mater., 2010, 22, 450-456.

4 L. Xie, X. Huang, C. Wu and P. Jiang, J. Mater. Chem., 2011, 21, 5897-5906.

5 Y. Song, Y. Shen, H. Liu, Y. Lin, M. Li and C.-W. Nan, J. Mater. Chem., 2012, 22, 16491-16498.

6 K. Hayashida and Y. Matsuoka, Carbon, 2013, 60, 506-513.

7 D. Wang, T. Zhou, J.-W. Zha, J. Zhao, C.-Y. Shi and Z.-M. Dang, J. Mater. Chem. A, 2013, 1, 6162-6168.

8 K. Brandt, C. Neusel, S. Behr and G. A. Schneider, J. Mater. Chem. C, 2013, 1, 3129-3137.

9 K. Hayashida, Y. Matsuoka and Y. Takatani, RSC Adv., 2014, 4, 33530-33536.
10 Y. Feng, W. L. Li, Y. F. Hou, Y. Yu, W. P. Cao, T. D. Zhang and W. D. Fei, J. Mater. Chem. C, 2015, 3, 1250-1260.

11 S. C. B. Mannfleld, B. C.-K. Tee, R. M. Stoltenberg, C. V. H.-H. Chen, S. Barman, B. V. O. Muir, A. N. Sokolov, C. Reese and Z. Bao, Nat. Mater., 2010, 9, 859-864.

12 C. Metzger and E. Fleisch, Appl. Phys. Lett., 2008, 92, 013506.

13 J. Li, J. Liang, L. Li, F. Ren, W. Hu, J. Li, S. Qi and Q. Pei, ACS Nano, 2014, 8, 12874-12882.

14 S.-J. Woo, J.-H. Kong, D.-G. Kim and J.-M. Kim, J. Mater. Chem. C, 2014, 2, 4415-4422.

15 B. Nie, R. Li, J. Cao, J. D. Brandt and T. Pan, Adv. Mater., 2015, 27, 6055-6062.

16 W. Hu, Z. Ren, J. Li, E. Askounis, Z. Xie and Q. Pei, Adv. Funct. Mater., 2015, 25, 4827-4836.

17 Y.-S. Chen, G.-W. Hsieh, S.-P. Chen, P.-Y. Tseng and C.-W. Wang, ACS Appl. Mater. Interfaces, 2015, 7, 45-50.

18 C. Kittel, in Introduction to Solid State Physics, ed. C. Kittel, John Wiley \& Sons, New York, 7th edn, 1996, ch. 8, pp. 197-232.

19 A. Schönhals and F. Kremer, in Broadband Dielectric Spectroscopy, ed. F. Kremer and A. Schönhals, Springer-Verlag, Berlin, 2003, ch. 3, pp. 59-98.

20 S. F. Wang and A. A. Ogale, Compos. Sci. Technol., 1993, 46, 93-103.

21 K. Hayashida and H. Tanaka, Adv. Funct. Mater., 2012, 22, 2338-2344.

22 K. Hayashida, RSC Adv., 2013, 3, 221-227.

23 K. Hayashida and Y. Matsuoka, Carbon, 2015, 85, 363-371.

24 A. Moezzi, A. M. McDonagh and M. B. Cortie, Chem. Eng. J., 2012, 185, 1-22.

25 A. Kolodziejczak-Radzimska and T. Jesionowski, Materials, 2014, 7, 2833-2881.

26 N. H. Langton and D. Matthews, Br. J. Appl. Phys., 1958, 9, 453-456.

27 K. Hayashida, H. Tanaka and O. Watanabe, Polymer, 2009, 50, 6228-6234.

28 Z.-M. Dang, L. Z. Fan, S.-J. Zhao and C.-W. Nan, Mater. Sci. Eng., B, 2003, 99, 386-389.

29 J. I. Hong, P. Winberg, L. S. Shadler and R. W. Siegel, Mater. Lett., 2005, 59, 473-476.

30 S. C. Tjong and G. D. Liang, Mater. Chem. Phys., 2006, 100, 1-5.

31 G.-S. Wang, Y. Deng, Y. Xiang and L. Guo, Adv. Funct. Mater., 2008, 18, 2584-2592.

32 H. Smaoui, L. E. L. Mir, H. Guermazi, S. Agnel and A. Toureille, J. Alloys Compd., 2009, 477, 316-321.

33 D. Ghosh, P. S. Sardar, M. Biswas, A. Mondal and N. Mukherjee, Mater. Chem. Phys., 2010, 123, 9-12.

34 G.-S. Wang, ACS Appl. Mater. Interfaces, 2010, 2, 1290-1293.

35 S. Vural, S. Koytepe, T. Seckin and I. Adiguzel, Mater. Res. Bull., 2011, 46, 1679-1685.

36 W. Wu, X. Huang, S. Li, P.-K. Jiang and T. Tanaka, J. Phys. Chem. C, 2012, 116, 24887-24895.

37 A. S. Roy, S. Gupta, S. Sindhu, A. Parveen and P. C. Ramanurthy, Composites, Part B, 2013, 47, 314-319.

38 N. Erdman, R. Campbeli and S. Asahina, Microsc. Today, 2006, 14, 22-25.

39 Y. U. Wang and D. Q. Tan, J. Appl. Phys., 2011, 109, 104102. 